\title{
Limits of the circles-in-the-sky searches in the determination of cosmic topology of nearly flat universes
}

\author{
G.I. Gomero, ${ }^{1}$ B. Mota, ${ }^{2}$ and M.J. Rebouças ${ }^{3}$ \\ ${ }^{1}$ Universidade Estadual de Santa Cruz - UESC, Rodovia Ilhéus - Itabuna $\mathrm{km} 16$, \\ 45650-000 Ilhéus - BA, Brazil \\ ${ }^{2}$ Instituto de Física, Universidade Federal do Rio de Janeiro , Av. Athos da Silveira Ramos 149, \\ Centro de Tecnologia - Bloco A - Cidade Universitria \\ 21941-972 Rio de Janeiro - RJ, Brazil \\ ${ }^{3}$ Centro Brasileiro de Pesquisas Físicas, Rua Dr. Xavier Sigaud 150, \\ 22290-180 Rio de Janeiro - RJ, Brazil
}

(Dated: June 1, 2022)

\begin{abstract}
An important observable signature of a detectable nontrivial spatial topology of the Universe is the presence in the cosmic microwave background sky of pairs of matching circles with the same distributions of temperature fluctuations — the so-called circles-in-the-sky. Most of the recent attempts to find these circles, including the ones undertaken by the Planck Collaboration, were restricted to antipodal or nearly antipodal circles with radii $\lambda \geq 15^{\circ}$. In the most general search, pairs of circles with deviation from antipodality angles $0^{\circ} \leq \theta \leq 169^{\circ}$ and radii $10^{\circ} \leq \lambda \leq 90^{\circ}$ were investigated. No statistically significant pairs of matching circles were found in the searches so far undertaken. Assuming that the negative result of general search can be confirmed through analysis made with data from Planck and future cosmic microwave background (CMB) experiments, we examine the question as to whether there are nearly flat universes with compact topology, satisfying Planck constraints on cosmological parameters, that would give rise to circles-in-the-sky whose observable parameters $\lambda$ and $\theta$ fall outside the parameter ranges covered by this general search. We derive the expressions for the deviation from antipodality and for the radius of the circles associated to a pair of elements $\left(\gamma, \gamma^{-1}\right)$ of the holonomy group $\Gamma$ which define the spatial section of any positively curved universe with a nontrivial compact topology. We show that there is a critical position that maximizes the deviation from antipodality, and prove that no matter how nearly flat the Universe is, it can always have a nontrivial spatial topology that gives rise to circles whose deviation from antipodality $\theta$ is larger than $169^{\circ}$, and whose radii of the circles $\lambda$ are smaller than $10^{\circ}$ for some observers's positions. This makes it apparent that slightly positively curved nearly flat universes with cosmological parameters within Planck bounds can be endowed with a nontrivial spatial topology with values of the observable parameters $\lambda$ and $\theta$ outside the ranges covered by the searches for circles carried out so far with either WMAP or Planck data. Thus, these circles-inthe-sky searches carried out so far are not sufficient to exclude the possibility of a universe with a detectable nontrivial cosmic topology. We present concrete examples of lens spaces universes whose associated circles have both, or at least one value of the observable parameters $(\lambda, \theta)$ outside the ranges covered by these searches. We also present a brief discussion of the implications of our results in view of unavoidable practical limits of the circles-in-the-sky method.
\end{abstract}

PACS numbers: 98.80.-k, 98.80.Es, 98.80.Jk

\section{INTRODUCTION}

Two fundamental problems regarding the FriedmannLemaître-Robertson-Walker (FLRW) approach to cosmological modeling concern the spatial geometry and topology. Regarding the geometry, recent high-precision cosmic microwave background (CMB) data from Planck have provided strong evidence that the universe is nearly flat with $\left|\Omega_{k}\right|<0.005$ [1], which is consistent with standard inflationary predictions that curvature should be unobservably small today. Concerning the topology, despite our present-day inability to predict it from a fundamental theory, one should be able to probe it through CMB observations (see, e.g., the reviews [2]).

An observable signature of a detectable nontrivial spatial topology is the presence in the CMB sky of pairs of matching correlated circles with equal distributions (up to a phase) of temperature fluctuations - patterns of hot and cold spots that match around the so-called circlesin-the-sky [3].

Each such pair of circles on the CMB sphere can be specified as a point in 6-dimensional parameter space, namely the center of each circle of the pair (four parameters), the angular radius of both circles (one parameter), and the relative phase between them (one parameter). Since such a general search for pairs of circles is very costly in computer time, most of recent searches, including the ones undertaken by the Planck team [4, 5], were restricted to back-to-back circle pairs ${ }^{1}$ [6-13] or nearly

\footnotetext{
1 This refers to pair of circles whose centers are antipodal points on the CMB sphere, which are also known as antipodal circlesin-the-sky.
} 
antipodal circles [6]. No pairs of matching circles were found through these searches. This negative result, along with the fact that in a very nearly flat $\left(\left|\Omega_{k}\right| \lesssim 10^{-5}\right)$ compact universe the deviation from antipodality is small for most observers [14, 15], has been taken to be sufficient to exclude a detectable nontrivial topology for most observers [15]. However, since the deviation from antipodality in compact orientable exactly flat $\left(\left|\Omega_{k}\right|=0\right)$ universes can be larger than 10 degrees [16], if the Universe is in fact flat then these restricted searches for antipodal or nearly antipodal circles would not be sufficient to rule out the possibility of a nontrivial flat topology [16].

Although the 6-dimensional parameter space can be used to statistically extract potential matching circles from CMB maps, it is not well adapted to study the relation between observable parameters of the circles and the spatial topology. In fact, while the circle radii and their relative phases are observable parameters directly linked to the topology, the positions of the circle centers depend on the choice of the coordinates and are related to the topology through the separation angle of circle centers $\Theta$, or equivalently its supplement $\theta$, which gives the deviation from antipodality. Thus, a more convenient set of parameters for studying the interrelations between matching circles and the spatial topology, includes these observable parameters directly linked to the topology, namely the deviation from antipodiciy, $\theta$, the relative phase angle, $\phi$, and the angular radii of the circles $\lambda$.

A more general search for circle pairs that are not backto-back has been carried out by Vaudrevange et al. [17] using Wilkinson Microwave Anisotropy Probe (WMAP) 7-years data [18]. No statistically significant pairs of matching circles were found. They have employed the circles-in-the-sky statistics to search for pairs of circles with radii $10^{\circ} \leq \lambda \leq 90^{\circ}$ and integer separation angles of the circle centers $11^{\circ} \leq \Theta \leq 180^{\circ}$, extending the existing lower bounds on these parameters so as to encompass a wider range of possible topologies. Thus, for example, the negative result of this search, if confirmed, along with the maximal values of the deviation from antipodality, $\theta_{\max }$, associated to the shortest geodesics in multiply-connected orientable flat manifolds [16], are sufficient to rule out the possibility of a detectable nontrivial orientable flat cosmic topology whose associated circle radii, $\lambda$, are greater than 10 degrees. ${ }^{2}$

Assuming that the negative result of the general search of Ref. 17] can be confirmed through a similar analysis made with data from Planck and future CMB experiments, an important remaining question that naturally arises here is whether there still are nearly flat, but not exactly flat, universes with compact topology that would

\footnotetext{
${ }^{2}$ In line with the usage in the literature, by the topology of the Universe, or simply cosmic topology, we mean the topology of its spatial sections.
}

give rise to circles-in-the-sky whose observable parameters $\lambda$ and $\theta$ would fall outside the parameter ranges covered by this more general search.

Our primary objective in this paper is to address this question by considering nearly flat universes whose spatial section $M$ is a slightly positively curved space $\left(\Omega_{k} \lesssim 0\right)$, whose cosmological parameters are within the bounds determined by Planck data [1], and endowed with a spherical orientable nontrivial topology. To this end, we first derive the analytic expressions for the deviation from antipodality and for the radius of the circles of any pair that arises from a general pair of elements $\left(\gamma, \gamma^{-1}\right)$ of the holonomy group $\Gamma$ used to form any quotient multiplyconnected spherical spaces $\mathbb{S}^{3} / \Gamma$. Second, for an arbitrary pair $\left(\gamma, \gamma^{-1}\right)$ of holonomies we derive an expression that gives the observer's position in which the deviation from antipodality attains its maximum. Third, we then show that no matter how nearly flat the Universe is it can always have a nontrivial topology that gives rise to an observable pair of matching circles whose deviation from antipodality $\theta$ is larger than $169^{\circ}$ (or $\Theta<11^{\circ}$ ) and with radii $\lambda$ smaller than $10^{\circ}$ for some observers. Therefore, with these observable parameters outside ranges covered by this general search [17], making clear that the circlesin-the-sky searches already undertaken are not sufficient to exclude the possibility of a detectable nontrivial topology for the Universe. Finally, by taking into account the recent bounds on the cosmological parameters by the Planck Collaboration [1], which constrain the distance to the last scattering surface $\chi_{\mathrm{LSS}}$, we concretely show examples of a number of lens spaces universes whose associated circles are such that the value of at least one of the observable parameters $(\lambda, \theta)$, or both, falls outside the range covered by the searches so far undertaken. These examples make apparent that it is possible to have a universe with a detectable nontrivial cosmic topology that respects Planck constraints on the cosmological parameters, and have not been excluded by the searches for circle-in-the-sky carried out so far.

The structure of the paper is as follows. In Section II we give a brief account of the prerequisites necessary for the following sections. In Section [I] we derive the expression for deviation from antipodality and for the radius of the circles associated to a pair of elements $\left(\gamma, \gamma^{-1}\right) \in \Gamma$ used to form the spatial sections of multiply-connected universes $\mathbb{S}^{3} / \Gamma$. In Section [IV] we show that no matter how small is the value for $\chi_{\text {LSS }}$ (in unit of curvature radius) there are always holonomies that give rise to observable circles-in-the-sky for which the maximal deviation from antipodality is detectable at some observer's position, and it can be made as close to $\pi$ as we require. In Section $\nabla$ we construct examples of universes with lensspace spatial topology whose values of cosmological parameters respect the bounds of Planck Collaboration [1], and for which both or at least one of the observable parameters of the circles $(\lambda, \theta)$ fall outside the range covered by the searches so far undertaken. In Sec. VI we present our final remarks and conclusions, and also briefly dis- 
cuss the implications of our results in view of unavoidable practical limits of the circles-in-the-sky method.

\section{PRELIMINARIES}

We begin by recalling the basic cosmological setting of this work. In the context of general relativity, a fundamental assumption in standard cosmological modelling is that, on large scales, the Universe is described by a 4dimensional manifold $\mathcal{M}=\mathbb{R} \times M$ endowed with the spatially homogeneous and isotropic FLRW spacetime metric

$$
d s^{2}=-c^{2} d t^{2}+a^{2}(t)\left[d \chi^{2}+f^{2}(\chi)\left(d \theta^{2}+\sin ^{2} \theta d \phi^{2}\right)\right]
$$

where $t$ is the cosmic time, $a(t)$ is the scale factor and $f(\chi)=(\chi, \sin \chi, \sinh \chi)$ depending on the sign of the constant spatial curvature $k=(0,1,-1)$.

The sections $M$ are often assumed to be the simplyconnected 3-dimensional manifolds: Euclidean $\mathbb{E}^{3}$, spherical $\mathbb{S}^{3}$, or hyperbolic $\mathbb{H}^{3}$. However, they can also be multiply-connected quotient 3-manifolds, which are quotient spaces $M=\widetilde{M} / \Gamma$, where the covering space $\widetilde{M}$ is the corresponding simply-connected constant curvature covering manifolds $\mathbb{E}^{3}, \mathbb{S}^{3}$ or $\mathbb{H}^{3}$, and $\Gamma$ is a discrete and fixed point-free group of isometries of $\widetilde{M}$ called the covering or holonomy group [19]. A generic element of the group $\Gamma$ is denoted by $\gamma$ and called holonomy or simply isometry.

Since we are concerned with nearly flat Universe whose spatial section is a slightly positively curved space $\left(\Omega_{k} \lesssim\right.$ $0)$ allowed by Planck constraints on cosmological parameter [1]), in the next sections we consider that the spatial section of the Universe can be modeled by any spherical orientable manifold with a nontrivial topology of the form $M=\mathbb{S}^{3} / \Gamma$.

Regarding the dynamics of the Universe we assume that it is given by a $\Lambda \mathrm{CDM}$ model, whose matter content is described by dust with density $\rho_{m}$, plus a cosmological constant $\Lambda$. The Friedmann equation can then be written in the form

$$
a^{2}=\frac{k c^{2}}{H^{2}(\Omega-1)}
$$

where $H=\dot{a} / a$ is the Hubble parameter, $\Omega=\Omega_{m}+\Omega_{\Lambda}$ with $\Omega_{m}=8 \pi G \rho_{m} / 3 H^{2}, \Omega_{\Lambda} \equiv 8 \pi G \rho_{\Lambda} / 3 H^{2}=\Lambda / 3 H^{2}$, and $G$ is the Newton's constant.

We recall that in the $\Lambda$ CDM setting one has that for a slightly positively curved nearly flat Universe the distance to the last scattering surface $\chi_{\mathrm{LSS}}$ in units of the curvature radius is given by ${ }^{3}$

$\chi_{\mathrm{LSS}}=\sqrt{\left|\Omega_{k 0}\right|} \int_{1}^{1+z_{\mathrm{LSS}}}\left[x^{3} \Omega_{m 0}+x^{2} \Omega_{k 0}+\Omega_{\Lambda 0}\right]^{-1 / 2} d x$,

where $\Omega_{k}=1-\Omega$ and the subscript 0 denotes evaluation at present time. Now, taking into account the recent bounds on the cosmological parameters by the Planck Collaboration [1] from Eq. (3) one has $\chi_{\mathrm{LSS}}=0.038$. We will use this value in the next sections to construct examples of nearly flat positively curved Universes with detectable nontrivial topology.

Another important ingredient we shall need in the following sections is the formulation of the conditions for detectability of cosmic topology. These conditions were studied for classes of hyperbolic and spherical manifolds as functions of the cosmological parameters in Refs. [20, 21], and extended to the case of generic manifolds in the inflationary limit in Ref. [15] (see also Refs. [14]). ${ }^{4}$ For this article, we only need to know that a way to study the detectability in cosmic topology is through the lengths of its closed geodesics as follows. The length of the closed geodesic generated by $\gamma \in \Gamma$ passing through a point $\mathbf{u} \in M$ is given by the distance between $\mathbf{u}$ and its image $\gamma \mathbf{u}$, i.e. by the distance function $d(\mathbf{u}, \gamma \mathbf{u})$ in the covering space. This allows the definition of the local injectivity radius $r_{i n j}(\mathbf{u})$ which is half the length of the smallest closed geodesic passing through the point $\mathbf{u}$. A necessary condition for detectability of cosmic topology is then given by

$$
r_{i n j}(\mathbf{u})<\chi_{\text {LSS }}
$$

where $\chi_{\mathrm{LSS}}$ is the comoving distance evaluated at $z_{\mathrm{LSS}}$. For universes whose nontrivial topology are globally homogeneous $r_{i n j}(\mathbf{u})$ is position-independent. However, for universes with globally inhomogeneous topology, the length of the smallest closed geodesic depends on the position $\mathbf{u}$. Therefore, to determine sufficient conditions for detectability that hold for all observers we have to use the global (constant) injectivity radius $r_{i n j} \equiv$ $\inf _{\mathbf{u} \in M} r_{i n j}(\mathbf{u})$, which is the radius of the smallest sphere inscribable in $M$.

To perform the calculations of the next sections we need the form of the holonomy transformations $\gamma$ as $4 \times 4$ matrices in $S O(4)$. We recall that the enumeration of all finite subgroups $\Gamma \in S O(4)$ can be made in terms of the much simpler enumeration of finite subgroups of $S O(3)$ (for a detailed account on this point we refer the readers to Ref. 24]). For the connection between $S O(4)$ and $S O(3)$ one uses quaternions, which are a generalization

\footnotetext{
3 Hereafter we express distances in units of the curvature radius $a_{0}=|k| H_{0}^{-1}\left|\Omega_{0}-1\right|^{-1 / 2}$, and measure angles in radians.

4 The question of whether the detection of a non-trivial cosmic topology can be used to set constraints on cosmological density parameters has been studied for particular topologies in Refs. 22] (see also the related Ref. [23]).
} 
of the familiar complex numbers with three imaginary unities $\mathbf{i}, \mathbf{j}$ and $\mathbf{k}$ satisfying the non-commutative multiplication rule

$$
\begin{array}{cc}
\mathbf{i}^{2}=\mathbf{j}^{2}=\mathbf{k}^{2}=-1, & \mathbf{i j}=-\mathbf{j i}=\mathbf{k} \\
\mathbf{j k}=-\mathbf{k} \mathbf{j}=\mathbf{i}, & \mathbf{k i}=-\mathbf{i k}=\mathbf{j} .
\end{array}
$$

Given a quaternion $\mathbf{q}=a \mathbf{1}+b \mathbf{i}+c \mathbf{j}+d \mathbf{k}$ with $(a, b, c, d) \in$ $\mathbb{R}^{4}$, one defines the conjugate quaternion as $\mathbf{q}^{*}=a \mathbf{1}-$ $b \mathbf{i}-c \mathbf{j}-d \mathbf{k}$ and the norm of $\mathbf{q}$ by $|\mathbf{q}|=\sqrt{\mathbf{q q}^{*}}=$ $\sqrt{a^{2}+b^{2}+c^{2}+d^{2}}$. Quaternions with norm 1 are called unit quaternions. For a unit quaternion one has an inverse given by $\mathbf{q}^{-1}=\mathbf{q}^{*}$. Geometrically, the set of all quaternions $\mathbf{q}=a \mathbf{1}+b \mathbf{i}+c \mathbf{j}+d \mathbf{k}$ defines the Euclidean space $\mathbb{E}^{4}$, while the 3 -sphere $\mathbb{S}^{3}$ can be identified with the multiplicative group of unit quaternions, i.e.

$$
\mathbb{S}^{3}=\{\mathbf{q} \in \mathbf{H} /|\mathbf{q}|=1\},
$$

where $\mathbf{H}$ denotes the associative quaternion algebra over the real numbers.

\section{DEVIATION FROM ANTIPODALITY AND RADII OF CIRCLES}

In this section we derive the analytic expressions for the deviation from antipodality and for the radius of the circles of any pair that arises from an arbitrary pair of elements $\left(\gamma, \gamma^{-1}\right)$ of the holonomy group $\Gamma$ used to form any quotient multiply-connected spherical spaces $\mathbb{S}^{3} / \Gamma$.

\section{A. Deviation from antipodality}

Without loss of generality, by a convenient choice of basis an arbitrary isometry $\gamma \in \Gamma$ can be written in the form [24]

$$
\gamma=\left[\begin{array}{cccc}
\cos \alpha & 0 & 0 & -\sin \alpha \\
0 & \cos \beta & -\sin \beta & 0 \\
0 & \sin \beta & \cos \beta & 0 \\
\sin \alpha & 0 & 0 & \cos \alpha
\end{array}\right]
$$

where $\alpha$ and $\beta$ are parameters used to define a generic isometry of $\mathbb{S}^{3}$. We recall that when the distance between a point $\mathbf{p} \in \mathbb{S}^{3}$ and its image $\gamma \mathbf{p} \in \mathbb{S}^{3}$ is independent of $\mathbf{p}$ the isometry $\gamma$ is called Clifford translation, which comes about if, and only if, $\alpha=\beta$.

For detectable topologies, the action of each pair of elements $\left(\gamma, \gamma^{-1}\right)$ of the group $\Gamma$ may generate one pair of matching circles in the CMB maps when the surface of last scattering (LSS) intersects its two images under the action of $\gamma$ and $\gamma^{-1}$. When $\gamma$ is a Clifford translation the pair $\left(\gamma, \gamma^{-1}\right)$ gives rise to a pair of antipodal matching circles. As we are interested in both back-toback and non-antipodal circles in nearly flat spherical universes with a non-trivial topology, we will focus on the general case $\alpha \neq \beta$, and also discuss specific instances of
Clifford translations. To calculate the distance between an observer and its $\gamma$-image we have to take into account the observer's position. ${ }^{5}$

To have a qualitative understanding of the calculation below it is useful to bear in mind the following three points. First, the distance between two points $\mathbf{u}$ and $\mathbf{v}$ in $\mathbb{S}^{3}$ is just the angle $\mu$ between them as seen from the origin of $\mathbb{E}^{4}$, and thus it is computed through the scalar product in $\mathbb{E}^{4}$ as $\langle\mathbf{u}, \mathbf{v}\rangle=\cos \mu$. Second, the line of sight of an observer at $\mathbf{u} \in \mathbb{S}^{3}$ looking at $\mathbf{v} \in \mathbb{S}^{3}$ is given by the tangent vector, at $\mathbf{u}$, to the geodesic joining these two points. Third, given three points $\mathbf{u}, \mathbf{v}_{1}$ and $\mathbf{v}_{2}$ in $\mathbb{S}^{3}$, the angular separation between $\mathbf{v}_{1}$ and $\mathbf{v}_{2}$ seen by an observer at $\mathbf{u}$ is the angle between the lines of sight of the observer at $\mathbf{u}$ looking at $\mathbf{v}_{1}$ and $\mathbf{v}_{2}$, and thus it is calculated through the scalar product of the corresponding tangent vectors at $\mathbf{u}$.

Now, let $\mathbf{w}_{1}$ and $\mathbf{w}_{2}$ be the centers of a pair of matched circles. As measured by the observer, these centers are given by their angular coordinates in the celestial (unit) sphere $\mathbb{S}^{2}$, so observationally the angle between these two points is calculated through their scalar product. However, since theoretical calculations are done in the 3 -sphere modelled as the set of unit quaternions, in this approach $\mathbf{w}_{1}$ and $\mathbf{w}_{2}$ are lines of sight between the observer and its images. So, they are represented as tangent vectors, at the observer's position, of the geodesics joining the observer with each of its images.

In Appendix A we present the detailed calculations showing that these tangent vectors are given by

$$
\mathbf{w}_{1}=\frac{1}{\sin \mu}\left(\mathbf{v}_{1}-\mathbf{u} \cos \mu\right) \text { and } \mathbf{w}_{2}=\frac{1}{\sin \mu}\left(\mathbf{v}_{2}-\mathbf{u} \cos \mu\right),
$$

where we have denoted the distance $d(\mathbf{u}, \gamma \mathbf{u})$ simply by $\mu$, for the sake of brevity. Equation (9) makes apparent that to have these tangent vectors we need to calculate the distance $\mu$. To compute this distance, which is nothing but $2 r_{i n j}(\mathbf{u})$, we first note that the holonomy $\gamma$ given by Eq. (8) consists of two independent rotations, namely a rotation of an angle $\alpha$ in the $\mathbf{x}_{1}-\mathbf{x}_{4}$ plane and a rotation of an angle $\beta$ in the $\mathbf{x}_{2}-\mathbf{x}_{3}$ plane. It follows that one can always choose the coordinate axes in $\mathbb{R}^{4}$ such that the observer is in the $\mathbf{x}_{1}-\mathbf{x}_{2}$ plane. In this way, to give the observer's position one needs only of one parameter, which is its distance to the axis $\mathbf{x}_{1}$. Denoting this distance by $\rho$ one has that the observer is given by

$$
\mathbf{u}=\cos \rho \mathbf{1}+\sin \rho \mathbf{i}
$$

The distance between the observer at $\mathbf{u}$ and its image

\footnotetext{
${ }^{5}$ Although the calculations carried out in the following hold for an arbitrary pair of holonomies $\left(\gamma, \gamma^{-1}\right)$, in dealing with detectable matching circles we focus on the holonomies $\gamma$ 's that generate the shortest closed geodesic passing through the observer's position $\mathbf{u} \in M$. Thus in these cases we have $d(\mathbf{u}, \gamma \mathbf{u})=2 r_{i n j}(\mathbf{u})$.
} 
$\mathbf{v}_{1}=\gamma \mathbf{u}$ is then given by

$$
\begin{aligned}
\cos \mu & =\langle\mathbf{u}, \gamma \mathbf{u}\rangle \\
& =\cos \alpha \cos ^{2} \rho+\cos \beta \sin ^{2} \rho .
\end{aligned}
$$

Clearly, the same expression holds for the distance between $\mathbf{u}$ and its image $\mathbf{v}_{2}=\gamma^{-1} \mathbf{u}$. Now, since we are interested in the deviation from antipodality, which is $\theta=\pi-\Theta$, where $\Theta$ is the angle between $\mathbf{v}_{1}$ and $\mathbf{v}_{2}$, a straightforward calculation whose details are given in Appendix C yields

$$
\begin{aligned}
\cos \theta & =-\left\langle\mathbf{w}_{1}, \mathbf{w}_{2}\right\rangle \\
& =1-\frac{2}{\sin ^{2} \mu}(\cos \alpha-\cos \mu)(\cos \mu-\cos \beta)(12
\end{aligned}
$$

for the deviation from antipodality of a pair of circles that arises from an arbitrary pair of holonomies $\left(\gamma, \gamma^{-1}\right)$ of the group $\Gamma$ used to form any generic quotient multiplyconnected spherical spaces $\mathbb{S}^{3} / \Gamma$.

It should be noted that the deviation from antipodality depends on the position of the observer given by $\rho$, since from Eq. (12) one clearly has that $\theta$ depends on $\mu$ (the distance between the observer and its $\gamma$-images), and from Eq. (11) $\mu$ depends on $\rho$. Moreover, it follows from Eq. (12) that the matched circles are antipodal $(\theta=0)$ for $\mu=\alpha$ and $\mu=\beta$; i.e., when the observer is at any of the two limiting positions $\rho=0$ or $\rho=\pi / 2$. For the observer's position in between these limiting values the matching correlated circles are not antipodal, and there are positions for which maximal deviations of antipodality come about.

In Appendix E we present detailed calculations showing that for an arbitrary pair of holonomies $\left(\gamma, \gamma^{-1}\right)$, the critical value $\mu_{0}$ for which the deviation from antipodality attains its maximum value is given by

$$
\cos \mu_{0}=\frac{1+\cos (\alpha+\beta)}{\cos \alpha+\cos \beta} .
$$

From this equation along with Eq. (12) we can derive the expression of the maximal deviation from antipodality for any given pair of holonomies, which is given by

$$
\theta_{\max }=|\beta-\alpha| .
$$

Finally, from Eqs. (11) and (13) one has that this maximal deviation from antipodality is detectable by an observer at a position $\rho_{0}$ given by

$$
\cos ^{2} \rho_{0}=\frac{\sin \beta}{\sin \alpha+\sin \beta}
$$

whenever the injectivity radius $r_{i n j}$ at $\rho_{0}$ is less than the distance to the LSS, i.e. whenever the necessary condition for detectability of cosmic topology $\mu_{0}<2 \chi_{\text {LSS }}$ is fulfilled at $\rho_{0}$.

\section{B. Angular radius of circles}

To derive the expression for the angular radius of a generic matching circle-in-the-sky that arises from an arbitrary pair of holonomies $\left(\gamma, \gamma^{-1}\right)$, we begin by recalling that $\chi_{\text {LSS }}$ denotes the comoving radius of the last scattering sphere.

To have a sketch of the calculations given in details below, let $\left[\mathbf{u}, \mathbf{v}_{1}\right]$ be the geodesic segment joining the observer and its image $\mathbf{v}_{1}=\gamma \mathbf{u}$, and let $\mathbf{p}$ be the middle point of this segment. Thus, a point $\mathbf{q}$ in the matching circle generated by the holonomy $\gamma$ lies in the geodesic plane orthogonal to $\left[\mathbf{u}, \mathbf{v}_{1}\right]$ at $\mathbf{p}$, and is distant $\chi_{\mathrm{LSS}}$ from $\mathbf{u}$ and $\mathbf{v}_{1}$. Now, given the segments of geodesics $[\mathbf{u}, \mathbf{p}]$ and $[\mathbf{u}, \mathbf{q}]$, one can easily find the tangent vectors $\mathbf{w}_{p}$ and $\mathbf{w}_{q}$ to these segments at the observer's position $\mathbf{u}$. The angular radius $\lambda$ of the matching circle is then given by the scalar product

$$
\cos \lambda=\left\langle\mathbf{w}_{p}, \mathbf{w}_{q}\right\rangle .
$$

To effectively compute the angular radius of matched circles it is convenient to choose a suitable coordinate system. In fact, without loss of generality the calculation can be carried out more easily by choosing a coordinate system such that the observer's position is $\mathbf{u}=\mathbf{1}$, and thus its image is

$$
\mathbf{v}_{1}=\gamma \mathbf{u}=\cos \mu \mathbf{1}+\sin \mu \mathbf{i} .
$$

The middle point of the geodesic segment $\left[\mathbf{u}, \mathbf{v}_{1}\right]$ is then

$$
\mathbf{p}=\cos \frac{\mu}{2} \mathbf{1}+\sin \frac{\mu}{2} \mathbf{i},
$$

and a generic point of the circle associated to the holonomy $\gamma$ has the form

$$
\mathbf{q}=t \mathbf{p}+y \mathbf{j}+z \mathbf{k},
$$

where $t, y$ and $z$ are real numbers subject to the conditions

$$
|\mathbf{q}|=1 \quad \text { and } \quad \cos \chi_{\mathrm{LSS}}=\langle\mathbf{1}, \mathbf{q}\rangle .
$$

The tangent vectors at $\mathbf{u}$ to the segments of geodesics $[\mathbf{u}, \mathbf{p}]$ and $[\mathbf{u}, \mathbf{q}]$ are then given, respectively, by

$$
\begin{aligned}
& \mathbf{w}_{p}=\frac{1}{\sin \frac{\mu}{2}}\left(\mathbf{p}-\cos \frac{\mu}{2} \mathbf{1}\right), \\
& \mathbf{w}_{q}=\frac{1}{\sin \chi_{\mathrm{LSS}}}\left(\mathbf{q}-\cos \chi_{\mathrm{LSS}} \mathbf{1}\right) .
\end{aligned}
$$

Substituting Eqs. (21) and (22) into Eq. (16), a simple calculation yields

$$
\cos \lambda=\frac{\tan (\mu / 2)}{\tan \chi_{\mathrm{LSS}}},
$$

for the angular radius of each matching circles generated by a general pair of holonomies $\left(\gamma, \gamma^{-1}\right)$ of the group 
$\Gamma$ used to form any multiply-connected spherical space $\mathbb{S}^{3} / \Gamma$.

Before proceeding to the next section, it is important to note that both, the deviation from antipodality and the radii of the circles-in-the sky, depend not only on the parameters $\alpha$ and $\beta$ that defines the holonomy $\gamma$, but also on the position of the observer determined by the parameter $\rho$ through the expression (11). This latter dependence, often neglected in the literature, will be of the utmost importance in the following sections.

\section{UPPER BOUND ON THE DEVIATION FROM ANTIPODALITY}

In this section we show that, for any observational value of $\chi_{\mathrm{LSS}}$, no matter how small it is, there are always holonomies that give rise to circles-in-the-sky for which the maximal deviation from antipodality $\theta_{\max }$ is detectable, and this deviation can be made as close to $\pi$ as we want. ${ }^{6}$

A general sketch of the proof is as follows. We begin by defining parameters $\epsilon$ and $\delta$ by the relations

$$
\frac{\beta+\alpha}{2}=\frac{\pi}{2}-\epsilon \quad \text { and } \quad \frac{\beta-\alpha}{2}=\frac{\pi}{2}-\delta,
$$

and rewriting Eqs. (13) and (15), respectively, as

$$
\cos \mu_{0}=\frac{\sin \epsilon}{\sin \delta} \quad \text { and } \quad \cos ^{2} \rho_{0}=\frac{\sin (\delta+\epsilon)}{2 \sin \delta \cos \epsilon} .
$$

We clearly have $\alpha=\delta-\epsilon$ and $\beta=\pi-(\delta+\epsilon)$, so the maximal deviation from antipodality given by Eq. (14) reduces to

$$
\theta_{\max }=\pi-2 \delta,
$$

which shows that a maximal deviation from antipodality close to $\pi$ requires a small value of $\delta$. On the other hand, the condition for detectability of cosmic topology at $\rho_{0}$ along with the near flatness of the universe is ensured, respectively, by the inequalities $\mu_{0}<2 \chi_{\text {LSS }} \ll 1$. Together with the first equation (25), these inequalities require that $\delta \approx \epsilon$, which in turn leads a very small value for $\alpha$. Since $\delta$ has a very small value, so $\operatorname{does} \epsilon$, and thus $\beta$ is very close to $\pi$.

To implement this general reasoning, consider the canonical generator of the lens space $L(p, q)$, with $p$ prime, given by Eq. (8) with

$$
\alpha=\frac{2 \pi}{p} \quad \text { and } \quad \beta=\frac{2 \pi q}{p} .
$$

Equations (24) and (27) yield

$$
\epsilon=\left[\frac{p-2(q+1)}{p}\right] \frac{\pi}{2} \text { and } \delta=\left[\frac{p-2(q-1)}{p}\right] \frac{\pi}{2},
$$

\footnotetext{
${ }^{6}$ Concrete examples of nearly flat spherical multiply-connected universe that illustrate this point are given in Sec. $\mathrm{V}$.
}

and thus

$$
\frac{\epsilon}{\delta}=1-\frac{4}{p-2(q-1)}
$$

Given any positive $\delta_{0} \ll 1$, which defines a maximal deviation from antipodality threshold $\theta_{0} \equiv \pi-2 \delta_{0}$, we will now show how to choose values for $p$ and $q$ such that $0<\epsilon<\delta<\delta_{0}$ and $\epsilon / \delta$ as close to 1 as we want $(\epsilon / \delta \approx 1)$ in such way that

$$
\cos \mu_{0}=\frac{\sin \epsilon}{\sin \delta}>\frac{\epsilon}{\delta}>\cos 2 \chi_{L S S} .
$$

Since this equation along with Eq. (26) imply that $\mu_{0}<$ $2 \chi_{\mathrm{LSS}}$ and $\pi>\theta_{\max }>\theta_{0}$, this amounts to saying that such nearly flat spherical universes could have the topology of a lens space with detectable maximal deviation from antipodality of matching circles as close to $\pi$ as we require.

To insure that $\epsilon / \delta>0$, from (29) one clearly has that $p-2(q-1)>4$, and thus

$$
p>2 q+2 .
$$

Hereafter, for each value of $p$, we shall refer to the values of $q>1$ satisfying this inequality as permissible values. ${ }^{7}$ The least impermissible value of $q$ is given by

$$
q_{0}=\frac{p-1}{2}
$$

since for $n=1,2,3, \ldots, q_{0}-2$, values of $q$ given by

$$
q_{n}=q_{0}-n
$$

satisfy Eq. (31) and so are all permissible values. Since $q_{n}>1$ for globally inhomogeneous spaces, from equations (32) and (33) it follows that $p>2 n+3$.

Substituting (32) and (33) into (28) one obtains

$$
\epsilon_{n}=\left(\frac{2 n-1}{p}\right) \frac{\pi}{2} \quad \text { and } \quad \delta_{n}=\left(\frac{2 n+3}{p}\right) \frac{\pi}{2},
$$

and thus

$$
\frac{\epsilon_{n}}{\delta_{n}}=\frac{2 n-1}{2 n+3} .
$$

Equation (35) shows that for a large enough value of $n$ we can have $\epsilon_{n} / \delta_{n} \approx 1$, and the second equation (34) shows that, additionally, for a large enough value $p$, we can have $\delta_{n}<\delta_{0}$, as we wanted to show.

Finally, we note that in the construction of the concrete examples of nearly flat multiply-connected universes we need a more precise estimate for the values of $n$ in terms

\footnotetext{
${ }^{7}$ Here, we do not allow $q=1$ since this corresponds to a Clifford translation, which gives rise to pairs of antipodal circles, i.e. $\theta=$ $0^{\circ}$.
} 
of $\chi_{\mathrm{LSS}}$. To this end, from Eq. (35) one has that the second inequality in (30), i.e. $\epsilon_{n} / \delta_{n}>\cos 2 \chi_{\text {LSS }}$, holds if

$$
n>\frac{1+3 \cos 2 \chi_{L S S}}{2\left(1-\cos 2 \chi_{L S S}\right)} .
$$

\section{CONCRETE EXAMPLES}

As a first illustrative example, consider a nearly flat universe with a lens-space spatial topology, and whose values of cosmological parameters respect the constraints on the cosmological parameters determined by Planck Collaboration [1] so that the distance to the LSS in units of the curvature radius is $\chi_{\mathrm{LSS}}=0.038$ [cf. Eq. (3)] Let us then look for a detectable holonomy that gives rise to circles-in-the-sky with detectable maximal deviation from antipodality larger than $99 \pi / 100$, which from Eq. (26) gives $\delta<\pi / 200$. Besides, from equation (36) one has that $n=692$ guarantees that $\epsilon_{n} / \delta_{n}>\cos 2 \chi_{\text {LSS }}$. Equations (34) in turn give

$$
\epsilon_{692}=\frac{1383 \pi}{2 p} \quad \text { and } \quad \delta_{692}=\frac{1387 \pi}{2 p} .
$$

Thus, to assure that $\delta_{692}<\pi / 200$ one has $p>138700$. Since we are looking for $p$ prime (this is not strictly necessary), we take the next prime greater than this value, i.e. $p=138727$ and obtain from Eq. (32) the least impermissible value of $q_{0}=69363$, and thus $q_{692}=68671$. In brief, this shows that in a nearly flat universe with $\chi_{\mathrm{LSS}}=0.038$, a maximal deviation from antipodality larger than $99 \pi / 100$ is detectable through the circles associated with the pair of holonomies $\left(\gamma, \gamma^{-1}\right)$ defined by Eq. (27), i.e. given by the canonical generator of the lens space $L(138727,68671)$. In fact, in this case, one has

$$
\theta_{\max }=\pi-2 \delta_{692} \approx 0.990002 \pi>\frac{99 \pi}{100} .
$$

In the following, we again take into account the Planck constraints on the cosmological density parameters [1] that permit $\chi_{\mathrm{LSS}}=0.038$, and present concrete examples (with smaller values for $p$ ) of nearly flat universes with lens-space spatial topology that give rise to detectable circles-in-the-sky in which either values of the observable parameters $\lambda$ (radius of circles) or $\theta$ (deviation from antipodality), or even both values, fall outside the ranges covered by the searches so far undertaken [6 13, 17].

In Table \ we collect together examples of nearly flat lens-space universes with $\chi_{\mathrm{LSS}}=0.038$ for which both parameters, the maximal deviation of antipodality, $\theta_{\max }$, and the radius of the matching circles, $\lambda$, fall outside the range covered by the most general search for circles [17]. For full details about the calculations for the construction of Table \we refer the readers to Appendix $\mathrm{G}$.

Although in all universes of Table \ both the radii of the circles, $\lambda$, and the deviation from antipodality, $\theta$, fall outside the ranges covered by the searches carried out so

\begin{tabular}{ccc}
\hline \hline$L\left(p, q^{\perp}\right)$ & $\mu_{0}$ & $\lambda$ \\
\hline$(24907,11763)$ & 0.075978 & $1.4^{\circ}$ \\
$(24943,11780)$ & 0.075923 & $2.6^{\circ}$ \\
$(24967,11791)$ & 0.075868 & $3.4^{\circ}$ \\
$(25013,11813)$ & 0.075814 & $4.0^{\circ}$ \\
$(25057,11834)$ & 0.075759 & $4.6^{\circ}$ \\
$(25087,11848)$ & 0.075705 & $5.1^{\circ}$ \\
$(25111,11859)$ & 0.075650 & $5.5^{\circ}$ \\
$(25147,11876)$ & 0.075596 & $5.9^{\circ}$ \\
$(25183,11893)$ & 0.075542 & $6.3^{\circ}$ \\
$(25219,11910)$ & 0.075488 & $6.7^{\circ}$ \\
\hline \hline
\end{tabular}

TABLE I: Globally inhomogeneous lens spaces $L(p, q)$ spatial topology of nearly flat universes with $\chi_{\mathrm{LSS}}=0.038$, for which both the maximal deviation of antipodality, $\theta_{\max }$, and the radius of the matching circles, $\lambda$, fall outside the range covered in the searches carried out with either WMAP or Planck data [6 13, 17]. As explained in Appendix G $q^{\perp}$ is the least value of $q$ for which $\theta_{\max }>170^{\circ}$, and $\mu_{0}$ is the critical value of the distance of the observer and its images for which the deviation from antipodality attains its maximum. The observer's position is given by $\rho_{0}$, which from Eq. (11) depends on $\mu_{0}$ for any given holonomy pair $\left(\gamma, \gamma^{-1}\right)$. In these universes $\theta_{\max } \approx 170^{\circ}$ for the observer at $\rho_{0}$ [cf. Eq. (25)].

far, clearly we only need that one of these parameters falls outside those ranges to have nearly flat universes with a nontrivial lens-space topology which has not been ruled out by the searches so far undertaken with either WMAP or Planck data [6 13, 17]. In Table [1] we collect examples of nearly flat universes (with $\chi_{\mathrm{LSS}}=0.038$ ) endowed with lens space spatial topology with detectable maximal deviation from antipodality $\theta_{\max } \gtrsim 90^{\circ}$, so within the range covered the most general search [17]. Nevertheless, with radii $\lambda<10^{\circ}$, so outside the range covered by these searches, and therefore not excluded by all searches so far undertaken. In Appendix $\mathrm{G}$ we give the full details of de calculations made for the construction of this Table.

Given that globally homogenous spaces give rise to antipodal circles $\left(\theta=0^{\circ}\right)$, an important remaining question that naturally arises here is whether there are nearly flat (with $\chi_{\text {LSS }}=0.038$ ) globally homogeneous lens-space universes that give rise to circles-in-the-sky with radii smaller than $10^{\circ}$, i.e. outside the range covered by the most general search so far undertaken [17]. In the remainder of this section we shall examine this question.

We begin by recalling that in globally homogeneous lens spaces $L(p, q)$ one has $q=1$, and that the injectivity radii $2 r_{i n j}(\mathbf{u}) \equiv \mu=d(\mathbf{u}, \gamma \mathbf{u})$ for these spaces are constants and given by $\mu=2 \pi / p$ 20]. Hence, equation (23) reduces to

$$
\cos \lambda=\frac{\tan \frac{\pi}{p}}{\tan \chi_{\mathrm{LSS}}} .
$$

Thus, in order to have a pair of matched circles with radius smaller than a certain value $\lambda_{0}$ (say) one must have

$$
p<\frac{\pi}{\arctan \left(\cos \lambda_{0} \tan \chi_{L S S}\right)}
$$




\begin{tabular}{ccc}
\hline \hline$L\left(p, q^{\perp}\right)$ & $\mu_{0}$ & $\lambda$ \\
\hline$(2203,552)$ & 0.075625 & $5.7^{\circ}$ \\
$(2207,553)$ & 0.075556 & $6.2^{\circ}$ \\
$(2213,555)$ & 0.075507 & $6.5^{\circ}$ \\
$(2221,557)$ & 0.075370 & $7.4^{\circ}$ \\
$(2237,561)$ & 0.075100 & $8.8^{\circ}$ \\
$(2239,561)$ & 0.075013 & $9.3^{\circ}$ \\
$(2243,562)$ & 0.074946 & $9.6^{\circ}$ \\
$(2251,565)$ & 0.074917 & $9.7^{\circ}$ \\
$(2267,572)$ & 0.074961 & $9.5^{\circ}$ \\
$(2269,572)$ & 0.074876 & $9.9^{\circ}$ \\
\hline \hline
\end{tabular}

TABLE II: Globally inhomogeneous lens spaces $L(p, q)$ spatial topology of nearly flat universes with $\chi_{\mathrm{LSS}}=0.038$, for which only the radii of the matching circles, $\lambda$, fall outside the range covered in the searches so far undertaken. As explained in Appendix G] $q^{\perp}$ is the least value of $q$ for which $\theta_{\max }>$ $90^{\circ}$, and $\mu_{0}$ is the critical value for which the deviation from antipodality attains its maximum. The observer's position is given by $\rho_{0}$ [cf. Eq. (25)], which from Eq. (11) depends on $\mu_{0}$ for any given holonomy pair $\left(\gamma, \gamma^{-1}\right)$. In these universes $\theta_{\max } \gtrsim 90^{\circ}$ for the observer at $\rho_{0}$. Note that the values of the parameters $p$ and $q$ are considerably smaller than those in Table I

which along with the condition for detectability of the topology, $\mu<2 \chi_{L S S}$, leads to

$$
p>\frac{\pi}{\chi_{L S S}} .
$$

For $\lambda_{0}=10^{\circ}$ and $\chi_{L S S}=0.038$ one obtains

$$
82.7<p<83.95 \text {. }
$$

Therefore, $\chi_{\mathrm{LSS}}=0.038$ allows for only one nearly flat globally homogeneous lens space universe that has not been ruled out by the searches so far undertaken, namely $L(83,1)$. This lens space universe would give rise to antipodal circles with radius smaller than $10^{\circ}$.

The extent to which the examples of this section depend upon the uncertainties in the determination of the cosmological parameters, which give rise to uncertainties on the determination of $\chi_{L S S}$, is an important point to be investigated, beyond the scope of the present article, though.

\section{CONCLUDING REMARKS}

The existence in the CMB anisotropy maps of correlated pairs of circles with the same distribution of temperature fluctuations (up to a phase), the so-called circles-in-the-sky, is a key prediction for a universe with a detectable non-trivial cosmic topology. Detecting such circles, and measuring the relative position of their centers, angular radii, and relative phase, would allow us to possibly determine the topology of the spatial section of the Universe.

Most of the recent searches, including the ones undertaken by the Planck Collaboration, were restricted to circles whose centers are antipodal points on the $\mathrm{CMB}$ sphere or nearly antipodal circles. In the most general search [17] the circles-in-the-sky statistics was employed to search for pairs of circles with radii $10^{\circ} \leq \lambda \leq 90^{\circ}$ and integer deviation from antipodality angles $0^{\circ} \leq \theta \leq 169^{\circ}$.

In this work, we have addressed the question as to whether there are still nearly flat slightly positively curved universes $\left(\Omega_{k} \lesssim 0\right)$, that respect Planck constraints on the cosmological parameters [1], and have compact spatial topology that would give rise to circles in CMB sphere whose observable parameters $\lambda$ (radius) and $\theta$ (deviation from antipodality) fall outside the parameter ranges covered by this general search.

To answer this question, we have derived the analytic expressions for the deviation from antipodality and for the radius of the circles that arise from a pair of elements $\left(\gamma, \gamma^{-1}\right)$ of the holonomy group $\Gamma$ which is used to define the spatial section of any positively curved universe with a nontrivial compact topology. Since the deviation from antipodality depends upon the observer's position $\rho$, we have derived the expression for the critical position $\rho_{0}$ that maximizes the deviation from antipodality in a universe with a generic $\mathbb{S}^{3} / \Gamma$ spatial topology. We then have shown that no matter how nearly flat the Universe is, it can always have a nontrivial topology whose deviation from antipodality $\theta$ of the associated pair of matching circles is larger than $169^{\circ}$ and whose radii $\lambda$ are smaller than $10^{\circ}$ for some observers's positions. This important result makes apparent that slightly positively curved nearly flat universes can always be endowed with a nontrivial spatial topology that gives rise to circles with two observable parameters associated to the topology, $\lambda$ and $\theta$, outside the range covered in the searches carried out so far with either WMAP and Planck data [6 -13, 17]. This makes clear that the circles-in-the-sky searches already undertaken are not sufficient to exclude the possibility of a universe with a detectable nontrivial cosmic topology, and that respects Planck constraints on the cosmological parameters.

By taking into account the recent constraints on the cosmological parameters by the Planck Collaboration [1], we have also exhibited concrete examples of lens spaces universes whose associated circles have both, or at least one value of the observable parameters $\lambda$ and $\theta$ outside the ranges covered by the searches so far undertaken. The existence of such concrete examples with very small radii of the circles, which are statistically difficult or even impossible to detect, has been conjectured in the recent Ref. [25].

Although only the limited range for deviation from antipodality $0^{\circ} \leq \theta \leq 169^{\circ}$ has been explored until now, the future searches for circles can in principle cover the whole range of values $0^{\circ} \leq \theta \lesssim 180^{\circ}$. However, regarding the radii of the circles, two facts should be taken into account. First, the peak amplitude in the circles statistic, $S^{\max }$, decreases with the radius of the circles due primarily to the Doppler term, which is increasing anticorrelated for circles with radius smaller than $45^{\circ}$ [6]. Sec- 
ond, the intersection of the peaks of the circle statistics with the false detection threshold line defines the smallest circle radius that one could expect to detect. Since the false positive line is higher for circles with smaller radius, in practice there is a minimum value for the radius of the circles we could expect to detect. The smaller value achieved in the search so far is around $\lambda \simeq 10^{\circ}$ with an estimated value $\lambda \simeq 5^{\circ}$ for future searches using polarized CMB maps 13]. The first five entries in Table \ are examples of nearly flat universes, respecting Planck constraints on the cosmological parameters, that give rise to matching circles with radii smaller than this minimum estimated value. For these lens spaces universes the unavoidable practical limits for small radii of the circles-in-sky method, due to the combination of the falling of the peaks amplitude with the increasing of the false detection threshold, are reached. Thus, the spatial topology of these universes cannot be unveiled through the circle-in-the-sky method.

\section{Acknowledgments}

M.J. Rebouças acknowledges the support of FAPERJ under a CNE E-26/102.328/2013 grant. M.J.R. also thanks Glenn Starkman for important discussions about the circles-in-the-sky searches, and $\mathrm{CNPq}$ for the grant under which this work was carried out. G.I.G. thanks the CBPF for kind hospitality during the first part of this work. We are also grateful to A.F.F. Teixeira for reading the manuscript and indicating some omissions and misprints.

\section{Appendix A}

\section{Appendix B: Tangent vectors}

In this appendix we compute the tangent vector at a point $\mathbf{u}$ on the 3 -sphere $\mathbb{S}^{3}$ of the geodesic joining $\mathbf{u}$ and another point $\mathbf{v}$ at a distance $\mu$.

Let $\mathbf{u}$ and $\mathbf{p}$ be two points in the 3 -sphere $\mathbb{S}^{3}$ such that the distance between them is $\pi / 2$. As shown in Ref. [26] (see Th. 2.1.5) the geodesic line from $\mathbf{u}$ to $\mathbf{p}$ is

$$
\mathbf{n}(s)=\mathbf{u} \cos s+\mathbf{p} \sin s .
$$

Since

$$
\langle\mathbf{n}, \mathbf{u}\rangle=\cos s,
$$

the geodesic is parametrized by the arc length $s$.

Let $\mathbf{v}$ be the point in this geodesic line located between $\mathbf{u}$ and $\mathbf{p}$ and at a distance $\mu$ from $\mathbf{u}$; then we have

$$
\mathbf{v}=\mathbf{u} \cos \mu+\mathbf{p} \sin \mu \text {. }
$$

We can use this expression to write the geodesic in terms of $\mathbf{u}$ and $\mathbf{v}$, in fact, we have

$$
\mathbf{p}=\frac{1}{\sin \mu}(\mathbf{v}-\mathbf{u} \cos \mu) \text {. }
$$

Substituting this expression into (B1) we obtain

$$
\mathbf{n}(s)=\frac{1}{\sin \mu}[\mathbf{u} \sin (\mu-s)+\mathbf{v} \sin s] .
$$

Taking the derivative at $s=0$ one gets the tangent vector pointing from $\mathbf{u}$ to $\mathbf{v}$,

$$
\mathbf{w}=\frac{1}{\sin \mu}(\mathbf{v}-\mathbf{u} \cos \mu) .
$$

\section{Appendix C}

\section{Appendix D: Deviation from antipodality $\theta$}

In this appendix we present details of the calculations of the deviation from antipodality $\theta$ given by equation (12).

Direct use of Eq. (9) and the fact that

$$
\left\langle\mathbf{u}, \mathbf{v}_{1}\right\rangle=\left\langle\mathbf{u}, \mathbf{v}_{2}\right\rangle=\cos \mu
$$

immediately yield

$$
\left\langle\mathbf{w}_{1}, \mathbf{w}_{2}\right\rangle=\frac{1}{\sin ^{2} \mu}\left(\left\langle\mathbf{v}_{1}, \mathbf{v}_{2}\right\rangle-\cos ^{2} \mu\right) .
$$

Now, since $\mathbf{v}_{1}=\gamma \mathbf{u}$ and $\mathbf{v}_{2}=\gamma^{-1} \mathbf{u}$, the very definition of isometry implies that $\left\langle\mathbf{v}_{2}, \mathbf{v}_{1}\right\rangle=\left\langle\mathbf{u}, \gamma^{2} \mathbf{u}\right\rangle$, and thus (8) and (10) imply that

$$
\left\langle\mathbf{v}_{1}, \mathbf{v}_{2}\right\rangle=\cos 2 \alpha \cos ^{2} \rho+\cos 2 \beta \sin ^{2} \rho .
$$

Moreover, from Eq. (11) it easily follows that

$$
\cos \alpha-\cos \beta=\frac{\cos \mu-\cos \beta}{\cos ^{2} \rho}=\frac{\cos \alpha-\cos \mu}{\sin ^{2} \rho},
$$

from which we obtain

$$
\cos ^{2} \rho=\frac{\cos \mu-\cos \beta}{\cos \alpha-\cos \beta} \quad \text { and } \quad \sin ^{2} \rho=\frac{\cos \alpha-\cos \mu}{\cos \alpha-\cos \beta} .
$$

Substituting these relations into Eq. (D3) one easily obtains

$$
\left\langle\mathbf{v}_{1}, \mathbf{v}_{2}\right\rangle=2(\cos \alpha+\cos \beta) \cos \mu-(2 \cos \alpha \cos \beta+1),
$$

which upon substitution into (D2) yields immediately equation (12).

\section{Appendix E}

\section{Appendix F: Critical distance $\mu_{0}$}

In this appendix we present details of the calculations of equation (13) that gives the critical distance $\mu_{0}$ which maximize the deviation from antipodality. 
Making the substitutions

$$
A=\cos \alpha \quad, \quad B=\cos \beta \quad \text { and } \quad x=\cos \mu
$$

into Eq.(12) it follows that, to minimize $\cos \theta$, i.e. maximize $\theta$, one has to maximize the function

$$
f(x)=\frac{(x-A)(x-B)}{x^{2}-1} .
$$

Since $\alpha$ and $\beta$ are never supplementary angles, i.e. they never sum up $\pi$, we in fact have $A+B \neq 0$.

Taking the first derivative $f^{\prime}(x)$ and equating the numerator to zero one obtains

$$
x^{2}-2 \ell x+1=0,
$$

where

$$
\ell=\frac{A B+1}{A+B} .
$$

Equation (F3) has real roots only if $\ell^{2} \geq 1$, which holds since $-1<A<1$ and $-1<B<1$. In fact, suppose first that $A+B>0$, since $(1-A)(1-B)>0$, then $\ell>1$. On the other hand, suppose that $A+B<0$, since $(1+A)(1+B)>0$, then $\ell<-1$. In either case the condition $\ell^{2} \geq 1$ is fulfilled.

Moreover, since $x \leq 1$, the solution to Eq. (F3) we are looking for is

$$
x_{0}=\ell-\sqrt{\ell^{2}-1},
$$

so, using back the substitutions (F1) and (F4), one gets

$$
\cos \mu_{0}=\frac{\cos \alpha \cos \beta+1}{\cos \alpha+\cos \beta}-\sqrt{\left(\frac{\cos \alpha \cos \beta+1}{\cos \alpha+\cos \beta}\right)^{2}-1} .
$$

To simplify this expression observe that

$$
(\cos \alpha \cos \beta+1)^{2}-(\cos \alpha+\cos \beta)^{2}=\sin ^{2} \alpha \sin ^{2} \beta,
$$

which, substituted into (F6) yields immediately (13).

\section{Appendix G}

\section{Appendix H: Construction of examples}

In this appendix we give full details of the calculations involved in the construction of concrete examples presented in Section $\mathrm{V}$.

From (25) and $\delta-\epsilon=\alpha=\frac{2 \pi}{p}$ we obtain

$$
\sin \frac{2 \pi}{p}=\left(\cos \frac{2 \pi}{p}-\cos \mu_{0}\right) \tan \delta .
$$

To look for models with maximal deviation from antipodality, $\theta_{\max }=\pi-2 \delta$, larger than a threshold $\theta_{0}$ it is enough to require

$$
\delta<\frac{\pi-\theta_{0}}{2}=\delta_{0},
$$

and thus, $\tan \delta<\tan \delta_{0}$. Recall now that in order to have observable matched circles we need $\cos \mu_{0}>\cos 2 \chi_{\mathrm{LSS}}$; thus using the fact that $\cos \frac{2 \pi}{p}<1$, together with the approximation $\sin \frac{2 \pi}{p} \approx \frac{2 \pi}{p}$, since $p \gg 1$, one obtains the inequality

$$
p>\frac{2 \pi}{\left(1-\cos 2 \chi_{\mathrm{LSS}}\right) \tan \delta_{0}} .
$$

Taking the threshold $\theta_{0}=170^{\circ}$, i.e. $\delta_{0}=\frac{\pi}{36}$ in radians, for $\chi_{\mathrm{LSS}}=0.038$ one obtains the lower bound $p>24879$. However, since we are restricting our analysis to prime values for $p$, this implies that $p \geq 24889$. This lower bound is very tight, since numerical calculations show that actually $p \geq 24907$, as shown in Tables II and III, which show examples of lens spaces with relatively small values of the parameter $p$ whose canonical generators have detectable maximal deviations from antipodality larger than $\theta_{0}=170^{\circ}$. Let us explain how these tables were built. We note that Tables II and IV are similarly constructed but correspond to a threshold $\theta_{0}=90^{\circ}$, i.e. $\delta_{0}=\pi / 4$ radians. Let us explain how these tables were constructed by referring to Tables [ and III]

In order to estimate efficiently the values for the parameter $q$ in Tables II and [II we recall the fact that all permissible values for $q$ are given by $q_{\eta}=q_{0}-n$, for $n=1,2,3, \ldots, q_{0}-2$, where $q_{0}=\frac{p-1}{2}$. Using equations (34) and ( $\mathrm{H} 2)$, for a maximal deviation from antipodality $\theta_{\max }>\theta_{0}$ one needs

$$
n<\frac{1}{2}\left[\left(1-\frac{\theta_{0}}{\pi}\right) p-3\right] .
$$

For the threshold $\theta_{0}=170^{\circ}$ one obtains

$$
n<\frac{1}{2}\left(\frac{p}{18}-3\right)
$$

which implies

$$
q_{n}>\frac{17 p}{36}+1
$$

The second column in Table $\amalg$ in calculated using this inequality.

Recalling that $\theta_{\max }=\pi-2 \delta$ and using (28) one obtains a simple expression to compute the maximal deviation from antipodality in terms of the parameters $p$ and $q$ of the lens space,

$$
\theta_{\max }=\frac{2(q-1) \pi}{p} .
$$

Note that for each value of $p$ there is a least value of $q$ for which inequality ( $[\mathrm{H} 6)$ holds, let $q^{\perp}$ be this least value. From (H7) one can see that for $q>q^{\perp}$ the maximal deviation of antipodality is $\theta_{\max }>\theta_{\max }^{\perp}>\theta_{0}$. For $q<q^{\perp}$, the maximal deviation from antipodality is $\theta_{\max }<\theta_{0}<\theta_{\max }^{\perp}$. The precise value of $\theta_{\max }$ is, however, very insensitive to the parameters $p$ and $q$; in fact, a 


\begin{tabular}{cccc}
\hline \hline$p$ & $q$ & $\mu_{0}$ & $\lambda$ \\
\hline 24907 & 11763 & 0.075978 & $1.4^{\circ}$ \\
\hline 24917 & 11768 & 0.075978 & $1.4^{\circ}$ \\
\hline 24919 & 11769 & 0.075978 & $1.4^{\circ}$ \\
\hline 24923 & 11771 & 0.075978 & $1.4^{\circ}$ \\
\hline 24943 & 11780 & 0.075923 & $2.6^{\circ}$ \\
& 11781 & 0.075979 & $1.4^{\circ}$ \\
\hline 24953 & 11785 & 0.075923 & $2.6^{\circ}$ \\
& 11786 & 0.075979 & $1.4^{\circ}$ \\
\hline 24967 & 11791 & 0.075868 & $3.4^{\circ}$ \\
& 11792 & 0.075923 & $2.6^{\circ}$ \\
& 11793 & 0.075979 & $1.4^{\circ}$ \\
\hline 24971 & 11793 & 0.075868 & $3.4^{\circ}$ \\
& 11794 & 0.075924 & $2.6^{\circ}$ \\
& 11795 & 0.075979 & $1.4^{\circ}$ \\
\hline 24977 & 11796 & 0.075868 & $3.4^{\circ}$ \\
& 11797 & 0.075924 & $2.6^{\circ}$ \\
& 11798 & 0.075979 & $1.4^{\circ}$ \\
\hline \hline
\end{tabular}

TABLE III: Globally inhomogeneous lens spaces $L(p, q)$, with $p$ prime, spatial topology of nearly flat universes with $\chi_{\mathrm{LSS}}=$ 0.038, for which both the maximal deviation of antipodality, $\theta_{\max }$, and the radius of the matching circles, $\lambda$, fall outside the range covered in the searches carried out so far. For each value of $p$ we display all the lens spaces $L(p, q)$ with the parameter $q$ in the interval $\left[q^{\perp}, q^{\top}\right]$, where $q^{\perp}$ is the least value of $q$ for which $\theta \max >170^{\circ}$, and $q^{\top}$ is the largest value of $q$ for which $\mu_{0}<\chi_{\mathrm{LSS}}$. In these universes $\theta_{\max } \approx 170^{\circ}$ for the observer at $\rho_{0}$ [cf. Eq. (25)].

direct calculation shows that the maximal deviation from antipodality for the spaces shown in Table III ranges between $\theta_{\max }=170.005^{\circ}$ and $\theta_{\max }=170.033^{\circ}$.

On the other hand, using equations (25) and (28) to write $\cos \mu_{0}$ in terms of the parameters $p$ and $q$, it is straightforward to check that the distance $\mu_{0}$ is an increasing function of $q$. So let $q^{\top}$ be the largest value of $q$ for which $\mu_{0}<2 \chi_{\mathrm{LSS}}$; i.e., for which the generator of $L(p, q)$ has detectable maximal deviation of antipodality. For $q>q^{\top}$ one has $\mu_{0}>2 \chi_{\text {LSS }}$, so that the maximal deviation from antipodality is not detectable. For $q<q^{\top}$, the maximal deviation from antipodality is detectable, however $\theta_{\max }<\theta_{\max }^{\top}$.

We observe numerically that the size of the interval $\left[q^{\perp}, q^{\top}\right]$ increases as $p$ increases. As one can see in Table
III, for values of $p$ from 24907 to 24923 one has $q^{\perp}=q^{\top}$, so there is only one lens space with detectable maximal deviation from antipodality larger than $\theta_{0}=170^{\circ}$. For $p=24943$ and $p=24953$ it holds $q^{\top}=q^{\perp}+1$, so for each of these values of $p$ there are only two lens spaces with detectable maximal deviation from antipodality larger than $\theta_{0}=170^{\circ}$. For the five primes between (and including) $p=24967$ and $p=24989$ it holds $q^{\top}=q^{\perp}+2$, so there are three such spaces; in Table III we show only those corresponding to the first three values of $p$. Note that, according to our analysis, larger values of $q$ correspond to smaller values of $\lambda$. In Table $\llbracket$ we show the lens spaces $L\left(p, q^{\perp}\right)$ corresponding to the first values of $p$ for which the size of the interval $\left[q^{\perp}, q^{\top}\right]$ increases in one unit.

\begin{tabular}{cccc}
\hline \hline$p$ & $q$ & $\mu_{0}$ & $\lambda$ \\
\hline 2203 & 552 & 0.075625 & $5.7^{\circ}$ \\
& 553 & 0.075733 & $4.8^{\circ}$ \\
& 554 & 0.075841 & $3.7^{\circ}$ \\
& 555 & 0.075949 & $2.1^{\circ}$ \\
\hline 2207 & 553 & 0.075556 & $6.2^{\circ}$ \\
& 554 & 0.075664 & $5.4^{\circ}$ \\
& 555 & 0.075771 & $4.4^{\circ}$ \\
& 556 & 0.075879 & $3.2^{\circ}$ \\
& 557 & 0.075987 & $2.1^{\circ}$ \\
\hline 2213 & 555 & 0.075507 & $6.5^{\circ}$ \\
& 556 & 0.075614 & $5.8^{\circ}$ \\
& 557 & 0.075721 & $4.9^{\circ}$ \\
& 558 & 0.075829 & $3.9^{\circ}$ \\
& 559 & 0.075937 & $2.3^{\circ}$ \\
\hline \hline
\end{tabular}

TABLE IV: Globally inhomogeneous lens spaces $L(p, q)$, with $p$ prime, spatial topology of nearly flat universes with $\chi_{\mathrm{LSS}}=$ 0.038 , for which the radii of the matching circles, $\lambda$, fall outside the range covered in the searches so far undertaken with either WMAP or Planck data. For each value of $p$ we display all the lens spaces $L(p, q)$ with the parameter $q$ in the interval $\left[q^{\perp}, q^{\top}\right]$, where $q^{\perp}$ is the least value of $q$ for which $\theta \max >90^{\circ}$, and $q^{\top}$ is the largest value of $q$ for which $\mu_{0}<\chi_{\text {LSS }}$. In these universes $\theta_{\max } \gtrsim 90^{\circ}$ for the observer at $\rho_{0}$. Note that the values of the parameters $p$ and $q$ are considerably smaller than those in Table III and that the lengths of the intervals $\left[q^{\perp}, q^{\top}\right]$ are correspondingly larger.
[1] P.A.R. Ade et al. (Planck Collaboration 2015), arXiv:1502.01589 [astro-ph.CO].

[2] G.F.R. Ellis, Gen. Rel. Grav. 2, 7 (1971); M. LachièzeRey and J.P. Luminet, Phys. Rep. 254, 135 (1995); G.D. Starkman, Class. Quantum Grav. 15, 2529 (1998); J. Levin, Phys. Rep. 365, 251 (2002); M.J. Rebouças and G.I. Gomero, Braz. J. Phys. 34, 1358 (2004); M.J. Rebouças, in Proc. XIth Brazilian School of Cosmology and Gravitation, eds. M. Novello and S.E. Perez Bergliaffa AIP Conference Proceedings vol. 782, p 188.

[3] N.J. Cornish, D. Spergel, and G. Starkman, Class. Quan- tum Grav. 15, 2657 (1998).

[4] P.A.R. Ade, et al. (Planck Collaboration 2013), Astron. Astrophys. 571, A26 (2014).

[5] P.A.R. Ade et al. (Planck Collaboration 2015), arXiv:1502.01593 [astro-ph.CO].

[6] N.J. Cornish, D.N. Spergel, G.D. Starkman, and E. Komatsu, Phys. Rev. Lett. 92, 201302 (2004).

[7] B.F. Roukema, B. Lew, M. Cechowska, A. Marecki, and S. Bajtlik, Astron. Astrophys. 423, 821 (2004).

[8] R. Aurich, S. Lustig, and F. Steiner, Class. Quantum Grav. 22, 2061 (2005). 
[9] R. Aurich, S. Lustig, and F. Steiner, Mon. Not. Roy. Astron. Soc. 369, 240 (2006).

[10] J.S. Key, N.J. Cornish, D.N. Spergel, and G.D. Starkman, Phys. Rev. D 75, 084034 (2007).

[11] P. Bielewicz and A.J. Banday, Mon. Not. R. Astron. Soc. 412, 2104 (2011).

[12] R. Aurich and S. Lustig, Mon. Not. R. Astron. Soc. 433, 2517 (2013).

[13] P. Bielewicz, A.J. Banday, and K.M. Gorski, Mon. Not. R. Astron. Soc. 421, 1064 (2012).

[14] B. Mota, G.I. Gomero, M.J. Rebouças, and R. Tavakol, Class. Quantum Grav. 21, 3361 (2004); B. Mota, M.J. Rebouças, and R. Tavakol, Int. J. Mod. Phys. A 20, 2415 (2005).

[15] B. Mota, M.J. Rebouças, and R. Tavakol, Phys. Rev. D. 78, 083521 (2008).

[16] B. Mota, M.J. Rebouças and R. Tavakol, Phys. Rev. D. 81, 103516 (2010).

[17] P.M. Vaudrevange, G.D. Starkman, N.J. Cornish, and D.N. Spergel, Phys. Rev. D 86, 083526 (2012).

[18] N. Jarosik et al., Astrophys. J. Suppl. Ser. 192,14 (2011).

[19] Thurston, W.P., Three-Dimensional Geometry and Topology. Vol.1, Edited by Silvio Levy, Princeton University Press (1997).

[20] G.I. Gomero, M.J. Rebouças, and R. Tavakol, Class. Quantum Grav.18, 4461 (2001).

[21] G.I. Gomero, M.J. Rebouças, and R. Tavakol,Class.
Quantum Grav. 18, L145 (2001); G.I.Gomero, M.J. Rebouças, and R. Tavakol, Int. J. Mod. Phys. A 17, 4261 (2002); B. Mota, M.J. Rebouças, and R. Tavakol, in Proc. of 10th Marcel Grossmann Meeting on General Relativity, edited by M. Novello, S.E. Perez Bergliaffa, R. Ruffini (World Sci. Publishing Co. Singapore 2005), Part C, pp. 1664-1667, arXiv:astro-ph/0403110

[22] M.J. Rebouças, J.S. Alcaniz, B. Mota, and M. Makler, Astron. Astrophys. 452, 803 (2006); M.J. Rebouças and J.S. Alcaniz, Mon. Not. Roy. Astron. 369, 1693 (2006); M.C. Bento, O. Bertolami, M.J. Rebouças, and P.T. Silva, Phys. Rev. D 73, 043504 (2006); M.C. Bento, O. Bertolami, M.J. Rebouças, and N.M.C. Santos, Phys. Rev. D 73, 103521 (2006); M.J. Rebouças and J.S. Alcaniz, Braz. J. Phys. 35, 1062 (2005); M.J. Rebouças, Int. J. Mod. Phys. D 16, 207 (2007); M.J. Rebouças, arXiv:astro-ph/0702428 and arXiv:astro-ph/0702642.

[23] B. Mota, M.J. Rebouças, and R. Tavakol, Phys. Rev. D. 84, 083507 (2011).

[24] E. Gausmann, R. Lehoucq, J.-P. Luminet, J.-P. Uzan, and J. Weeks, Class. Quantum Grav. 18, 5155 (2001).

[25] J.P. Luminet, Universe 2, 1 (2016). arXiv:1601.03884 [astro-ph.CO]

[26] J.G. Ratcliffe, Foundations of Hyperbolic Manifolds, Graduate Texts in Mathematics 149, 2nd Edition, Springer (2006). 